\title{
Handelbare Umweltrechte als Steuerungs- instrument für den Straßengüterverkehr in sensiblen Regionen
}

Fallbeispiel Brennerautobahn

\section{Tradeable permits as a steering instrument for goods traffic in ecologically sensitive areas}

The case of the Brenner motorway

\section{Kurzfassung}

Die Zunahme der Verkehrsnachfrage in Europa und die damit verbundenen steigenden ökonomischen und ökologischen Belastungen sind Ursache für die wiederkehrende Diskussion hinsichtlich einer nachhaltig orientierten Verkehrspolitik. In diesem Rahmen wird auch der Einsatz neuer verkehrspolitischer Instrumente erwogen. Vor diesem Hintergrund untersucht der Beitrag, inwieweit handelbare Umweltrechte eine Alternative zum bisher eingesetzten Instrumentenmix darstellen. Hierzu wurde ein System handelbarer Umweltrechte für den Straßengüterverkehr in ökologisch sensiblen Regionen konzipiert und für einen alpinen Verkehrskorridor auf theoretischer Ebene implementiert. Die resultierenden Auswirkungen auf die Verkehrs- und Umweltbelastungen wurden mithilfe von eigens entwickelten Instrumenten untersucht. Die gewonnenen Schlussfolgerungen können als Basis für eine kritische Bewertung des Einsatzes eines solchen Systems herangezogen werden.

\begin{abstract}
The continuous growth of traffic volume in Europe over the past years and the corresponding economic and ecological impacts are the reasons for a recurring discussion of a more sustainability-oriented transport policy. In this context, the implementation of new transport policy instruments is part of the discussion. Against this background, the article investigates to what extent tradeable permits can be an alternative to the existing mix of transport policy instruments. A system of tradeable permits for heavy goods vehicles in ecologically sensitive areas has been designed and subsequently implemented on a theoretical level for one of the alpine corridors. The impact on traffic volume and the environment have been analysed using ad-hoc models. Conclusions drawn from these models can be used as a basis for a future application of such a system.
\end{abstract}




\section{Einleitung}

Seit Beginn der 90er Jahre wird in der Europäischen Union über eine "Strategie für eine dauerhaft umweltgerechte Mobilität“ (KOM 92/46) nachgedacht. Vor dem Hintergrund eines kontinuierlichen Verkehrswachstums, verbunden mit steigenden Belastungen für Mensch und Umwelt, wurde so der ökologische Aspekt in die Diskussion einer neuen, verursachergerechten Verkehrspolitik integriert. In diesem Sinn regte das 1995 von der Europäischen Kommission vorgelegte Grünbuch „Faire und effiziente Preise im Verkehr" (KOM 95/691) eine Internalisierung der externen Kosten des Verkehrs an. Als geeignete Internalisierungsinstrumente werden für gewöhnlich sowohl Steuern als auch Straßenbenutzungsgebühren betrachtet. Derartige reine Monetarisierungsansätze mindern jedoch kaum die unweigerlich durch die Überlastung europäischer Hauptverkehrsadern entstehenden Umweltschäden.

Handelbare Umweltrechte hingegen sorgen über die klare Definition von Umweltzielen für eine strikte Einhaltung festgelegter Schadstoffgrenzwerte. Überdies führen sie zu einer kosteneffizienten Verteilung der immer knapper werdenden Ressource Verkehrsinfrastruktur. Daher kann ihr Einsatz im Straßenverkehr, insbesondere in hochgradig exponierten, sensiblen und schutzbedürftigen Gebieten wie Küsten- oder Bergregionen, gegenüber prominenten Konzepten als vielversprechend betrachtet werden.

Vor diesem Hintergrund wurde untersucht, ob und in welchem Maße handelbare Umweltrechte eine Alternative zum bisher eingesetzten verkehrspolitischen Instrumentenmix darstellen. Hierzu wurde zunächst ein System handelbarer Umweltrechte für ökologisch besonders sensible Regionen entworfen. Dabei wurde speziell der Straßengüterverkehr betrachtet, der in signifikantem Maß für verkehrsbedingte Schadstoff- und Lärmbelastungen verantwortlich ist. In einem zweiten Schritt wurden die vermutlichen Auswirkungen dieses Systems auf die Verkehrs- und Umweltbelastungen in einem Modellraum simuliert, und zwar am Beispiel des alpinen Verkehrskorridors Brennerautobahn, der als ökologisch besonders sensibler und verkehrsbedingt hoch belasteter Wirkungsraum gilt. Für diese Wirkungsanalyse am Modellfall wurden wiederum eigens Instrumente entwickelt.

\section{Die Internalisierung der externen Effekte des Verkehrs}

Die Kapazität vieler Hauptverkehrsachsen stößt in den letzten Jahren zunehmend an ihre Grenzen - und das langjährige Credo europäischer Verkehrspolitik, der steigenden Nachfrage mit einem steigenden Angebot zu begegnen, wird letzten Endes in Frage gestellt. Zwischen 1991 und 2001 stieg das Straßengüterverkehrsaufkommen in der EU15-Zone um $38 \%$, gemessen in tkm (vgl. Europäische Kommission 2003). Da sich analog zur steigenden Verkehrsnachfrage außerdem die umwelt- und gesundheitsschädlichen Belastungen durch verkehrsbedingte Schadstoffemissionen potenziert haben, wird vermehrt eine Internalisierung der externen Kosten des Verkehrs gefordert. Im Folgenden werden daher kurz die theoretischen Grundlagen einer solchen Internalisierung sowie die externen Kosten des Verkehrs vorgestellt. Im Anschluss daran erfolgt eine Präsentation gängiger verkehrspolitischer Internalisierungsinstrumente.

\section{Die Theorie der Internalisierung}

Nach der Theorie der Wohlfahrtsökonomik erfolgt in der Marktwirtschaft eine optimale Allokation der Ressourcen über den Marktpreis, der sämtliche KostenNutzen-Abwägungen widerspiegelt. Die Ressourcen stehen denjenigen zur Verfügung, für die deren Nutzen größer oder gleich den Kosten, also dem zu zahlenden Preis ist (vgl. Geisendorf 1994). Somit kann der Marktpreis als Maß für die Knappheit einer Ressource betrachtet werden und steuert dessen Allokation (vgl. Schütte 1998).

Ende des 18. Jahrhunderts legte der Ökonom Marshall dar, dass die optimale Ressourcenallokation häufig durch das Vorhandensein von „external economies“ verhindert wird. Nach Endres et al. (1998) bestehen solche „externen Effekte“ darin, dass die Nutzensituation eines Individuums unmittelbar von einer Aktivität abhängt, die von einem anderen Individuum kontrolliert wird. Grundsätzlich können positive und negative externe Effekte unterschieden werden: Positive Effekte sind Vorteile, die Wirtschaftssubjekte durch die Aktivität anderer Wirtschaftssubjekte haben. Unter negativen externen Effekten werden Nachteile durch die Aktivität anderer verstanden. Führt man eine monetäre Bewertung dieser Nachteile durch, können die sog. ,externen Kosten" quantifiziert werden (vgl. Bickel et al. 1994). Aufbauend auf den Ideen von Marshall forderte Pigou 1920 in seinem Werk "The Economics of Welfare“, dass die negativen externen Effekte dem Verursacher angelastet werden sollen. Eine solche monetäre Anlastung wird allgemein als Internalisierung externer Effekte bezeichnet. 


\section{Die externen Kosten des Verkehrs}

Jede Verkehrsleistung umfasst Nutzen und Kosten, die jedoch nicht vollständig vom Nutzer der Verkehrsinfrastruktur bezahlt, sondern anderen Personen bzw. der Gesellschaft angelastet werden. Sie bilden die Differenz zwischen privaten und sozialen Grenzkosten (vgl. Schütte 1998). Interne oder private Kosten sind die Kosten, die der Nutzer für seine Fahrt trägt. Hierzu gehören typischerweise der Zeitaufwand, Anschaffungs- und Betriebsausgaben für das Fahrzeug, Straßenbenutzungsgebühren sowie persönlich getragene Unfallfolgekosten. In der von der Forschungsgemeinschaft INFRAS (Zürich) und dem Institut für Wirtschaftspolitik und Wirtschaftsforschung (IWW, Karlsruhe) wiederholt durchgeführten Studie „External Costs of Transport“ (Basisjahre: 1990, 1995, 2000) wurden folgende, nicht durch den Verursacher getragenen negativen externen Effekte identifiziert und einer monetären Bewertung unterzogen:

\section{- Lärm}

Lärm verursacht Kosten sowohl in Form von Wertminderungen der Grundștücke als auch durch Schädigung der menschlichen Gesundheit. Bei einem Wert von über $65 \mathrm{~dB}(\mathrm{~A})$ erhöht sich nachweislich das Risiko von Herz-Kreislauferkrankungen um bis zu 20\%. Zudem kann bereits ein Lärmwert von $30 \mathrm{~dB}(\mathrm{~A})$ zu Schlafstörungen führen (vgl. Troge 1993).

\section{- Luftschadstoffe}

Luftverschmutzungen durch Stickoxide ( $\mathrm{NO}_{\mathrm{x}}$ ), Kohlenmonoxid ( $\mathrm{CO})$, Schwefeldioxid $\left(\mathrm{SO}_{2}\right)$, flüchtige organische Verbindungen (VOC) sowie durch Staub- und Dieselpartikel (PM) verursachen gleichermaßen Schäden an der menschlichen Gesundheit, an Gebäuden als auch an der Biosphäre (vgl. INFRAS et al. 2000). $\mathrm{NO}_{\mathrm{x}}$ sowie Partikel gelten als die gesundheitsschädlichsten externen Effekte des Verkehrs.
Neben Lärm und Luftschadstoffen wurden in die Bewertung außerdem Auswirkungen des Verkehrs auf die Bereiche "Natur und Landschaft", „Unfälle", „Staueffekte“, "Zerschneidung städtischer Gebiete sowie Kosten der Raumknappheit“ sowie „Kosten durch Up- and Downstream-Prozesse" einbezogen.

Abbildung 1 gibt einen Überblick über die in dieser Studie für das Jahr 2000 in der EU15 (unter Einbeziehung der Schweiz und Norwegen) ermittelten externen Kosten. Sie verdeutlicht, dass gerade der Straßengüterverkehr, nicht zuletzt aufgrund seines bedeutenden Anstiegs in den letzten Jahrzehnten, maßgeblich für lokale Belastungen, beispielsweise durch Luftschadstoffe, verantwortlich gemacht werden kann.

\section{Abbildung 1}

Externe Kosten des Straßenverkehrs - 2000

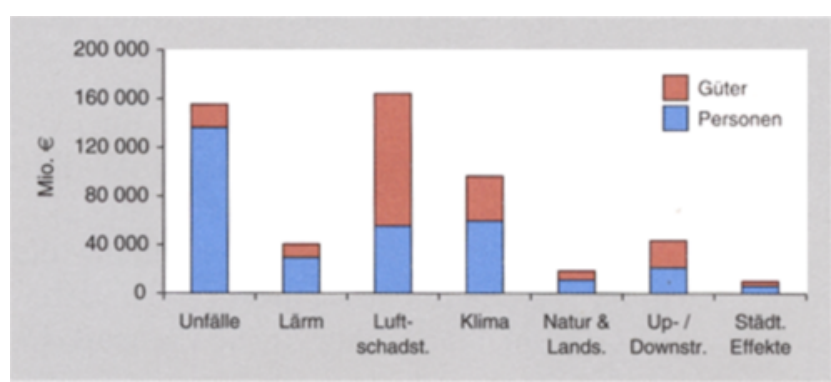

Quelle: INFRAS et al. 2004

\section{Internalisierungsinstrumente der externen Kosten des Verkehrs}

Im Straßenverkehr ermöglichen derzeit Steuern (Mineralöl- und Kraftfahrzeugsteuer) und Straßenbenutzungsgebühren eine annähernd verursachergerechte Internalisierung der externen Effekte. Jedoch weisen beide Instrumente einige nicht unbedeutende Schwächen auf (Übersicht 1).

Übersicht 1

Nachteile herkömmlicher verkehrspolitischer Internalisierungsinstrumente

\begin{tabular}{|l|l|}
\hline Steuern & Straßenbenutzungsgebühren \\
\hline $\begin{array}{l}\text { Nur begrenzt ökologisch treffsicher } \\
\text { (nur ein Teil der externen Kosten wird berücksichtigt) }\end{array}$ & $\begin{array}{l}\text { Mengenwirkung unsicher } \\
\text { (geringe Preiselastizität des Verkehrs) }\end{array}$ \\
$\begin{array}{l}\text { Internationale Wettbewerbsverzerrungen } \\
\text { (verursacht durch unterschiedlich hohe nationale Steuern) } \\
\text { Mengenwirkung unsicher } \\
\text { (geringe Preiselastizität des Verkehrs) } \\
\begin{array}{l}\text { Informationsproblem } \\
\text { (ideale Höhe unbekannt) }\end{array}\end{array}$ & \begin{tabular}{l} 
(ideale Gebührenhöhe unbekannt) \\
\hline
\end{tabular}
\end{tabular}


Erforderlich sind neue verkehrspolitische Instrumente, die einerseits Angebot und Nachfrage bei der Verkehrsinfrastruktur in Einklang bringen und gleichzeitig die durch den Straßenverkehr verursachten Schädigungen limitieren. Handelbare Umweltrechte zeichnen sich, wie im nächsten Abschnitt dargelegt, durch genau diese Merkmale aus.

\section{Handelbare Umweltrechte und ihr Einsatz im Straßenverkehr}

Handelbare Umweltrechte sind verbriefte Rechte, die es dem Halter gestatten, die Umwelt in einem bestimmten Maße zu schädigen. Das Maß der als erträglich erachteten Schädigung wird durch die Menge emittierter Rechte determiniert. Die Entwicklung der ersten theoretischen Modelle handelbarer Umweltrechte geht auf die Ideen von Coase (Coase Theorem) und Dales in den 1960er Jahren zurück. Praktisch fanden Umweltrechte seitdem vor allem in den USA in vielfältiger Art und Weise Anwendung. In Europa zählen Umweltrechte dagegen erst seit der Einführung des Europäischen Emissionshandelssystems zum umweltpolitischen Inventar.

Im Gegensatz $\mathrm{zu}$ anderen ökonomischen umweltpolitischen Instrumenten wie Umweltsteuern, die ihr Ziel ausschließlich durch Verteuerung des Preises der Verschmutzung erreichen, wird bei Umweltrechten eine kosteneffiziente Verteilung der knappen Ressource durch den Rechtehandel erreicht. Der Preis für das knappe Gut Umweltverschmutzung wird also nicht exogen vorgegeben, sondern auf dem Markt ermittelt. Handelbare Umweltrechte stellen somit ein umweltpolitisches Instrument dar, welches ein hoheitlich festgelegtes Umweltziel auf ökonomische und kosteneffiziente Weise erreicht.

Ein erster Versuch der praktischen Umsetzung eines Umweltrechtesystems (ohne Handel) im Straßengüterverkehr wurde mit der Einführung des Ökopunktesystems 1993 in Österreich durchgeführt. Ziel war es, mit der Ausgabe sog. Ökopunkte die im österreichischen Alpentransitverkehr emittierten umweltschädlichen Emissionen nachhaltig zu reduzieren. Dem gemäss wurde jährlich eine bestimmte Anzahl an Ökopunkten, der maximal tragbaren Schadstoffbelastung entsprechend, emittiert und nach einem vorher festgelegten Schlüssel an alpenüberquerende Staaten verteilt. Ziel war eine Reduktion der Stickoxid-Belastungen $\left(\mathrm{NO}_{\mathrm{x}}\right)$ um $60 \%$ bis zum Jahr 2003. Allerdings scheiterte dieses System am politischen Widerstand.

\section{Handelbare Umweltrechte im Straßengüterverkehr ökologisch sensibler Regionen - Entwurf eines Systems}

Im Folgenden wird das von Anke Eßer (2004, Diplomarbeit) entworfene System handelbarer Umweltrechte für den Straßengüterverkehr in ökologisch besonders sensiblen Regionen vorgestellt. Ziel dieser Arbeit war es, den neuen Anforderungen an eine verursachergerechte ökologische Verkehrspolitik gerecht zu werden. Besonderer Wert wurde dabei auf eine adäquate räumliche und zeitliche Differenzierung des Handelssystems sowie eine entsprechende Bemessungsgrundlage gelegt. Das System versucht, die Grundlagen für einen transparenten und liquiden Markt (vgl. Hansjürgens et al. 2003) zu schaffen, eine kontinuierliche Preisentwicklung $\mathrm{zu}$ fördern und Marktdefizite wie Transaktionskosten zu verhindern, um eine kosteneffektive Verteilung der Rechte (vgl. Baumol et al. 1988) zu ermöglichen. Seine wesentlichen Merkmale sind in Übersicht 2 dargestellt und werden im Folgenden in ihren Grundprinzipien näher ausgeführt, dabei abschließend gegenüber bestehenden Systemen von Straßenbenutzungsgebühren abgegrenzt.

\section{Räumliche Differenzierung des Umweltrechtesystems}

Die räumliche Differenzierung eines Systems handelbarer Umweltrechte betrifft sowohl die Auswahl der betroffenen Gebiete als auch der Straßen, auf denen die Rechte erhoben werden. Entsprechend der in den Kraetzschmer et al. (2004) gegebenen Definition für sensible Regionen können die wichtigsten alpenquerenden Transitstrecken aufgrund ihrer spezifischen Bedingungen als Gebiete mit einer verstärkten Sensibilität bezüglich verkehrsbedingter Belastungen betrachtet werden. Um in diesen Gebieten ein Ausweichen des Verkehrs auf das sekundäre Straßennetz zu vermeiden, sollte ein Fahrverbot für rechtepflichtige Lkws außerhalb von Autobahnen verhängt werden. Zur Vorbeugung einer Diskriminierung lokal ansässiger Unternehmen bieten sich kostenlose Sonderzuteilungen von Umweltrechten oder zweckgebundene Finanztransfers an diese Unternehmen an (vgl. Koschel et al. 1998). Da finanzielle Mittel aus einem zweckgebundenen Finanztransfer neben dem Kauf benötigter Umweltrechte auch zur Investition in schadstoffärmere Lkw genutzt werden können, erscheint diese Alternative für das hier vorgestellte Umweltrechtesystem am geeignetsten. 


\section{Übersicht 2}

Das System handelbarer Umweltrechte für den Straßengüterverkehr in ökologisch besonders sensiblen Regionen

\begin{tabular}{|c|c|}
\hline Rechtehalter & Transportunternehmer \\
\hline Betroffene Fahrzeuge & Alle Fahrzeuge mit einem zulässigen Gesamtgewicht ab 2,2 Tonnen \\
\hline Bemessungsgrundlage & $\mathrm{NO}_{x^{\prime}}, \mathrm{PM}_{10}$ und Lärm \\
\hline $\begin{array}{l}\text { Betroffene Gebiete } \\
\text { (hier berücksichtigte Gebiete) }\end{array}$ & Alpenkorridore: Brenner, Gotthard, Fréjus, Mont Blanc und Tauern \\
\hline Betroffene Straßen & Transitautobahnen, Fahrverbot für Lkw auf sekundärem Straßennetz \\
\hline Emissionszeitpfad & Abhängig von saisonaler Schadstoffkonzentration und Verkehrsaufkommen (vgl. Abb. 2) \\
\hline Lokale Unternehmen & Erhalten finanzielle, zweckgebundene Ausgleichszahlungen \\
\hline Sonstiges & Bestehende Nacht- sowie Wochenendfahrverbote bleiben erhalten \\
\hline \multicolumn{2}{|l|}{ Merkmale des Handelssysteme } \\
\hline Abrechnung der Rechte & Sofortige Abrechnung über GPS und Mobilfunktechnik während der Fahrt \\
\hline „Banking“ und „Borrowing“ & Nicht erlaubt \\
\hline Marktteilnehmer & Transportunternehmen und rechteemittierende Instanz \\
\hline Primärverteilung & $\begin{array}{l}\text { Vor Handelsbeginn: Mittels „Sealed Bid Uniform Price“ Auktionen } \\
\text { Nach Handelsbeginn: Im Rahmen der Sekundärverteilung }\end{array}$ \\
\hline Sekundärverteilung & $\begin{array}{l}\text { Öber Onlinebörse, an welcher der „Market Clearing Price“ nach dem Kassakurs- } \\
\text { Prinzip bestimmt wird }\end{array}$ \\
\hline Gültigkeit der Rechte & 30 Tage \\
\hline Veröffentlichung der Ausgabemengen & $\begin{array}{l}\text { Langfristig: Frühzeitige Veröffentlichung des Reduktionsziels } \\
\text { Mittelfristig: Monatliche Mengen werden } 12 \text { Monate im Voraus veröffentlicht } \\
\text { Kurzfristig: Wöchentliche Mengen werden relativ kurzfristig veröffentlicht, da sie } \\
\text { als Feinsteuerungsinstrument dienen }\end{array}$ \\
\hline Ausgabezyklus & 7 Tage \\
\hline Minimalpreis & Wird zur Deckung der Wege-/Infrastrukturkosten festgelegt \\
\hline Verwendung der Einnahmen & Entscheidung muss auf politischer Ebene getroffen werden \\
\hline Struktur der Umweltagentur & $\begin{array}{l}\text { Behörde: Obernimmt administrative Aufgaben } \\
\text { Kommission: Öbernimmt notwendige politische Entscheidungen und } \\
\text { Steuerungsaufgaben }\end{array}$ \\
\hline
\end{tabular}

Quelle: Eßer 2004

\section{Bemessungsgrundlage und Rechtehalter}

Die Bemessungsgrundlage bestimmt, „warum“ die Rechte erhoben werden. Für ein System handelbarer Umweltrechte in ökologisch besonders sensiblen Regionen erscheint eine Kombination verschiedener Schadstoffe im Hinblick auf die ökologische Treffsicherheit des Systems am zweckmäßigsten. Da sich die Bemessungsgrundlage nach den lokalen Belastungssituationen im Wirkungsgebiet der Rechte, also entlang der Alpenkorridore richtet, wurden Stickoxide, Staubpartikel und Lärm für das hier vorgestellte System ausgewählt. Sie wurden nach Ausmaß der Belastung durch den jeweiligen Schadstoff gewichtet und auf einen Kilometer normiert. So wurde festgelegt, wie viele Rechte ein Lkw pro Kilometer bei einer Alpenüberquerung zu entrichten hat (vgl. Tabelle 1).
Tabelle 1

Anzahl der Rechte pro Kilometer nach Fahrzeugklassen

\begin{tabular}{|l|c|}
\hline Euro 1 & $2,53($ Rechte $/ \mathrm{km})$ \\
Euro 2 & $1,56($ Rechte $/ \mathrm{km})$ \\
Euro 3 & $1($ Recht $/ \mathrm{km})$ \\
Euro 4 & $0,56($ Rechte $/ \mathrm{km})$ \\
Euro 5 & $0,55($ Rechte $/ \mathrm{km})$ \\
\hline
\end{tabular}

Die Wahl der Rechtehalter kann sowohl „upstream“ als auch „downstream“ erfolgen. Bei $\mathrm{CO}_{2}$-Emissionsrechten im Energiesektor beispielsweise wären Brennstofferzeuger die „Upstream"- und Endverbraucher die „Downstream"-Rechtehalter. Als ideale Rechtehalter erscheinen hier die Transportunternehmen ("downstream", direkte Emission), da sie zum einen über die genausten Informationen bezüglich des gesamten Transportbedarfs verfügen und zum anderen für eine möglichst effiziente Auslastung und Organisation mit dem kombinierten Verkehr sorgen können. 


\section{Zeitliche Differenzierung der Umweltrechteemission}

Bezüglich der temporalen Gestaltung des Umweltrechtesystems gilt es zu definieren, „wann" bzw. „wann wie viele" Rechte emittiert werden. Dabei muss die Emission von Umweltrechten derart gestaltet sein, dass Planungsunsicherheiten auf Seiten der Transportunternehmer vermieden werden. Entscheidende Kriterien zur Bestimmung des Emissionszeitpunkts und der Emissionsmenge sind die zeitliche Verteilung der Transportnachfrage und der Schadstoffbelastungen. Abbildung 2 stellt einen zeitlichen Vergleich zwischen saisonalen Schwankungen der Frachtraumnachfrage und Partikelemissionen in einem ausgewählten Raum dar.

\section{Abbildung 2}

Verteilung der Frachtraumnachfrage und der Partikelemission

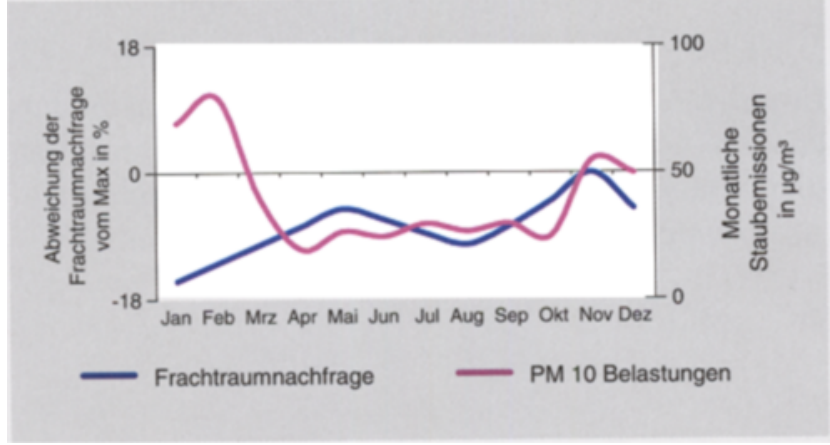

Quelle: Lebek 2003; Bundesamt für Umwelt, Wald und Landschaft (BUWAL) 2001

Da die zeitliche Verteilung der Frachtraumnachfrage sowie die Belastungskurven zum Teil gegenläufige Entwicklungen aufweisen, muss ein Kompromiss bezüglich des Zeitpfades der Umweltrechteemission gefunden werden. Einen Vorschlag für eine entsprechende qualitative Gestaltung des Zeitpfades enthält Abbildung 3 .

\section{Abbildung 3}

Qualitativer Zeitpfad der Umweltrechtemissionen

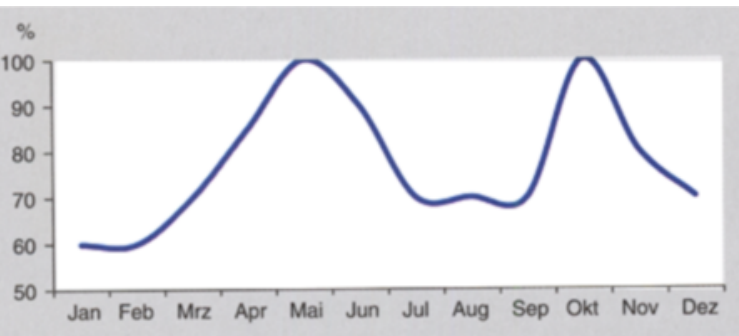

Quelle: Lebek 2003; BUWAL. 2001

Die Mengen zulässiger Emissionen sollten langfristig kontinuierlich abnehmen und die Ausgabemengen wöchentlich definiert werden. Zudem ist es zur Ge- währleistung der nötigen Planungssicherheit wichtig, die mittelfristig (monatlich oder quartalsmäßig) ausgegebenen Mengen frühzeitig zu veröffentlichen, beispielsweise ein Jahr im Voraus. Die wöchentlichen Ausgabemengen dienen indes der Feinsteuerung des Systems.

\section{Der Handel mit Umweltrechten}

Der Handel mit Umweltrechten kann bilateral, also durch direkte Absprache der Handelspartner, über Makler („Over-the-Counter“) oder über Börsen abgewickelt werden. Die Ausgabe der Umweltrechte erfolgt entweder durch kostenlose Verteilung („Grandfathering"), mittels Auktionen oder durch Verkauf zu Festpreisen.

In dem hier vorgestellten System handelbarer Umweltechte erfolgt die erste Verteilung der Rechte vor Handelsbeginn mittels "Sealed Bid Uniform Price“Auktionen, die sich durch die Abgabe geschlossener Gebote und eine umsatzmaximierende Preisfestlegung auszeichnen. Im weiteren Verlauf werden marginale Mengen an Umweltrechten im Rahmen der Sekundärverteilung emittiert. Die Sekundärverteilung, also der Handel mit Umweltrechten, wird über eine Onlinebörse abgewickelt, an der der Marktpreis nach dem Kassakurs-Prinzip (periodisch, basierend auf vorliegenden Aufträgen) bestimmt wird.

\section{Handelbare Umweltrechte und Wegekosten}

Handelbare Umweltrechte sollten andere ordnungspolitische Instrumente entweder vollständig ersetzen oder adäquat ergänzen. Konflikte in Wirkung und Zielsetzung treten hierbei insbesondere in Bezug auf die in sensiblen Gebieten erhobenen Straßenbenutzungsgebühren auf. Die rechtliche Grundlage für die Erhebung der Straßenbenutzungsgebühren bildet in der Europäischen Union die "Wegekostenrichtlinie" (Richtlinie 1999/62/EG) und in der Schweiz das Landesverkehrsabkommen.

Beide rechtlichen Grundlagen berechtigen - die geplante Änderung der "Wegekostenrichtlinie" vorausgesetzt - zur Erhebung erhöhter Gebühren in besonders sensiblen Gebieten und rechtfertigen so indirekt auch eine über die Infrastrukturkosten hinausgehende Kostenbelastung durch handelbare Umweltrechte. Der Tatbestand erhöhter finanzieller Belastungen sowie deren Grundlage ist bei Straßenbenutzungsgebühren und Umweltrechten identisch. Sie unterscheiden sich lediglich in der Art und Weise der Preisermittlung. Da eine gleichzeitige Erhebung von Straßenbenutzungsgebühren und Umweltrechten in den betroffenen Gebie- 
ten jedoch zu einer doppelten Belastung führen würde, werden in dem hier vorgestellten System die Kosten der Infrastrukturbereitstellung über einen Mindestpreis integriert. Der über diesen Mindestpreis hinausgehende Abgabepreis der Umweltrechte repräsentiert dann die Gebühren für exzessive Umweltbelastungen in den betroffenen Gebieten.

\section{Simulation: Auswirkungen des Systems am Beispiel der Brennerautobahn}

Die Auswirkungen der Einführung des Systems handelbarer Umweltrechte für den Straßengüterverkehr werden im Folgenden anhand der sensiblen Region um die Brennerautorbahn simuliert. Sie betreffen durch die Veränderung der Rahmenbedingungen sowie durch die implizite Reduzierung der Transitnachfrage zum einen den Wettbewerb im Straßengüterverkehr. Zum anderen werden unweigerlich Veränderungen im Verkehrsaufkommen und in der Umweltbelastung der betroffenen Gebiete hervorgerufen.

\section{Instrumente zur Implementierung und Bewertung}

Das Bewertungsinstrument, das hier zur Analyse der Auswirkungen verwendet wird, besteht aus folgenden Einzelmodulen (vgl. Kraetzschmer et al. 2004):
- Fernverkehrsmodell VACLAV (vgl. Schoch 2004)

Das europäische Fernverkehrsmodell stellt Informationen bezüglich Verkehrsbelastungen, Entfernungen und Transportzeiten bereit. Das Modell wurde am IWW entwickelt und in verschiedenen nationalen und internationalen Forschungsprojekten als Prognoseinstrument eingesetzt (z.B. in TEN-STAC 2004).

\section{- ASTRA-T (vgl. Schade 2004)}

Das ASTRA-T-Modul beschreibt die volkswirtschaftlichen Auswirkungen der Szenarien, u.a. auf das Bruttoinlandsprodukt und die Beschäftigung. Das Flottenmodul „vehicle fleet module (VFM)“ stellt einen Teil des makroökonomischen ASTRA-T-Modells dar und ermöglicht unter anderem eine zeitreihenbasierte Berechnung von Flottenstrukturdaten.

- Regio-SUSTAIN (vgl. Schmedding et al. 2005)

Unter Verwendung dieses Immissionsanalysemoduls können Schadstoff- und Lärmbelastungen ermittelt und anhand einer zuvor auf GIS-Basis ermittelten Bevölkerungsverteilung monetär bewertet werden.

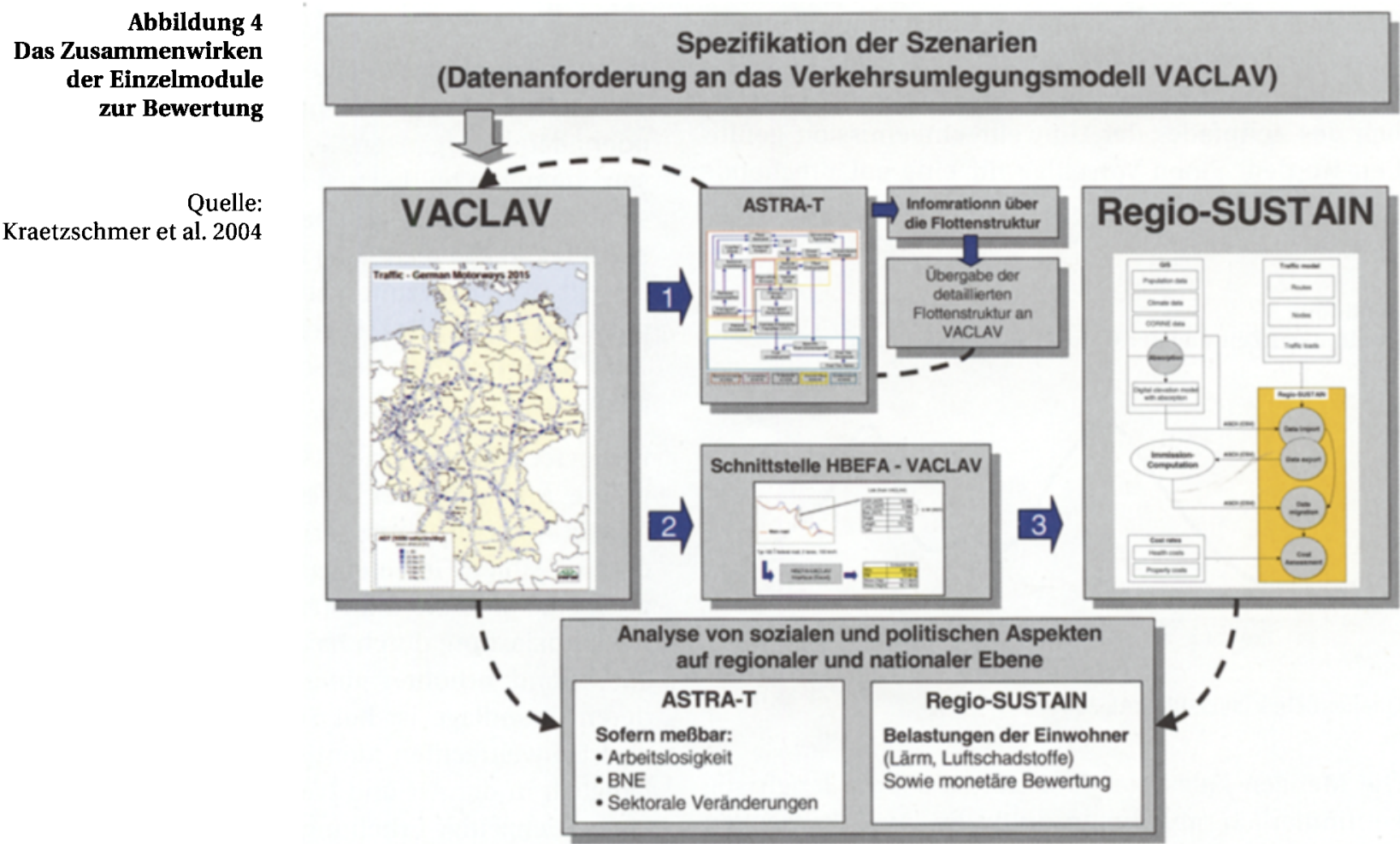


- HBEFA-VACLAV (vgl. Schmedding et al. 2005)

Diese Schnittstelle zwischen der Emissionsdatenbank (HBEFA: Handbuch für Emissionsfaktoren, hrsg. vom Umweltbundesamt) und dem Verkehrsmodell erlaubt die Berechnung verkehrsbedingter Emissionsmengen.

Abbildung 4 gibt einen Überblick über die Verflechtung zwischen den eingesetzten Modellen. Jedoch wurde im Folgenden aufgrund des Umfangs auf die Untersuchung der einzelnen Szenarien hinsichtlich der politischen und sozialen Auswirkungen sowie auf die Berechnung der externen Kosten verzichtet (vgl. Kraetzschmer et al. 2004).

Für die Beispielregion und die unterschiedlichen Szenarien wurden zunächst über das Transportmodell VACLAV die Verkehrsbelastungen der betroffenen Straßen berechnet. Zusätzlich zu den bereits in VACLAV bereitgestellten Informationen bezüglich Transportentfernungen, -zeiten und Fahrzeugzahlen wurden hierzu die durch ASTRA-T ermittelten Angaben bezüglich der Flottenstruktur verwendet (vgl. Kraetzschmer et al. 2004). Das Modul Regio-SUSTAIN ermöglicht die Untersuchung der Szenarien hinsichtlich Wirkung auf Mensch und Umwelt.

\section{Vorbereitung der Simulation}

In diesem Abschnitt werden die einzelnen Schritte zur Vorbereitung und Durchführung der Simulation dargestellt. In diesem Rahmen wird zuerst die getroffene Auswahl der Beispielregion begründet, bevor anschließend die zu betrachtenden Szenarien definiert werden.

\section{- Auswahl der Beispielregion}

Aufgrund ihrer besonderen topographischen und klimatischen Eigenschaften können die Alpen als ökologisch besonders sensible Region betrachtet werden. Zudem sind vor allem die Haupttransitstrecken St. Gotthard-, Fréjus-, Mt. Blanc- und Tauerntunnel sowie Brennerpass in hohem Maße verkehrsbedingten Schadstoff- und Lärmbelastungen ausgesetzt. Um eventuelle Ausweichreaktionen des Güterfernverkehrs einschätzen zu können, wäre es sicherlich wünschenswert, den gesamten Alpenraum bzw. zumindest alle in Frage kommenden Transitstrecken in die Analyse einzubeziehen. Dies ist jedoch aufgrund der Komplexität und der hohen Anforderungen an die einzelnen zum Einsatz kommenden Modelle derzeit nur schwer möglich. Daher wurde die Analyse repräsentativ auf die Brennerautobahn (zwischen Innsbruck und Brennersee) beschränkt. In dem Untersuchungsraum mit einer Gesamtlänge von $35 \mathrm{~km}$ und $4 \mathrm{~km}$ Breite leben insgesamt 138139 Personen.

\section{- Szenarien}

Entsprechend dem Ziel der Fallstudie, die kurz- wie auch langfristigen Effekte der Einführung eines Systems handelbarer Umweltrechte für den Straßengüterverkehr in sensiblen Regionen nachzubilden, wurden folgende drei Szenarien festgelegt und für diese im Rahmen der Simulation mögliche Änderungen des Verkehrsaufkommens und der Belastungen von Mensch und Umwelt durch Stickoxide, Dieselrußpartikel und Lärm analysiert:

(1) Basisszenario I: Belastungen 2004 ohne Umwelt rechte

(2) Szenario II: Belastungen 2004 mit Umweltrechten

(3) Szenario III: Belastungen 2020

mit Umweltrechten.

\section{Die Auswirkungen bei Einführung \\ des Umweltrechtesystems}

Das entworfene System handelbarer Umweltrechte für den Straßengüterverkehr in sensiblen Regionen hat zum Ziel, durch eine Begrenzung der Menge emittierter Umweltrechte die Anzahl der Fahrten durch den betrachteten Brennerkorridor zu kontingentieren. Entsprechend ist nach der Einführung eine Reduzierung der Verkehrsbelastung sowie der Umweltbelastungen durch Lärm, Stickoxide und Dieselrußpartikel zu erwarten.

Die Vorbereitung der Simulation umfasst neben der Festlegung der Beispielregion und der Szenarien überdies die Definition des mit der Einführung des Umweltrechtesystems angestrebten Umweltziels. Dieses wird in einem System handelbarer Umweltrechte über die Menge der ausgegebenen Rechte zum Ausdruck gebracht. Zudem sind bei der vom Verkehrsumlegungsmodell durchgeführten Netzwerkoptimierung die Kosten der Brennerüberquerung von Bedeutung, da im Modell diese Kosten den Widerständen auf einzelnen Straßenabschnitten entsprechen.

\section{- Das Reduktionsziel}

Das Reduktionsziel in einem System handelbarer Umweltrechte wird über die Menge der emittierten Umweltrechte festgelegt. Die Zahl der gestatteten Transitfahrten lässt sich daraus über die Anzahl der benötigten Rechte in Abhängigkeit vom Umweltstandard der Fahrzeuge (Euronormen) ableiten.

Im Rahmen der Euronormfestlegung bis 2008 (Euro 5) wurde eine Reduktion der Stickoxidemissionen auf $2 \mathrm{~g} /$ $\mathrm{kWh}$ und für Partikel auf $0,02 \mathrm{~g} / \mathrm{kWh}$ festgelegt. Dies 
entspricht im Vergleich zur heute gültigen Euro-Norm 3 einer Reduktion um $60 \%$ beziehungsweise $80 \%$ (vgl. Abgasgrenzwerte, hrsg. vom Landesanstalt für Umweltschutz Baden Württemberg). Das vom Umweltbundesamt deklarierte Reduktionsziel fordert, die Lärmbelastung bis 2010 tagsüber auf $59 \mathrm{~dB}(\mathrm{~A})$ und nachts auf $49 \mathrm{~dB}(\mathrm{~A})$ zu senken (vgl. Gühnemann 1999).

Für die Simulation wurde eine Reduktion der Stickoxidemission um $70 \%$, der Partikelemission um $90 \%$ und eine Stabilisierung der Lärmemission auf $80 \mathrm{~dB}(\mathrm{~A})$ bis 2020 definiert. Hieraus lassen sich die durchschnittlich für ein Fahrzeug pro Brennerüberquerung benötigten Rechte ableiten. Für das Jahr 2004 wurde im Vergleich zum Jahr 2003 mit gezählten 1574000 Lkws zunächst von einer leicht reduzierten Anzahl von Transitfahrten in Höhe von 1403000 Fahrten von Fahrzeugen der Euro-Norm 3 ausgegangen. Tabelle 2 gibt eine Übersicht über die jeweils zur Verfügung stehenden Umweltrechte.

Tabelle 2

Anzahl ausgegebener Umweltrechte

\begin{tabular}{|l|c|c|}
\hline & $\mathbf{2 0 0 4}$ & $\mathbf{2 0 2 0}$ \\
\hline $\begin{array}{l}\text { Anzahl emittierter } \\
\text { Umweltrechte pro Jahr }\end{array}$ & 87000000 & 23000000 \\
\hline $\begin{array}{l}\text { Anzahl Lkw } \\
\text { Transitfahrten pro Jahr }\end{array}$ & 1403000 & 1405000 \\
\hline $\begin{array}{l}\text { Anzahl Lkw } \\
\text { Transitfahrten pro Tag }\end{array}$ & 3843 & 3849 \\
\hline
\end{tabular}

\section{- Auswirkungen auf die Verkehrssituation}

Mit Hilfe des Verkehrsumlegungsmodells VACLAV konnten sowohl die aktuelle Situation (2004) wie auch die Szenarien mit den implementierten Umweltrechten für die Jahre 2004 und 2020 berechnet werden. Abbildung 5 stellt die ermittelten Verkehrsbelastungen (DTV) an der Brennerautobahn A13 (Raststätte Matrei) dar. Für die aktuelle Situation (2004) ohne Umweltrechte (UR) ist danach eine tägliche Belastung von 7868 Lkws zu erwarten. Dies würde eine Erhöhung des LkwVerkehrsaufkommens am Brenner um mehr als $3555^{2}$ Lkws täglich im Vergleich zu 2003 bedeuten.

Ziel war, das Lkw-Aufkommen durch die Einführung der Umweltrechte bis 2004 bzw. 2020 auf 3843 Lkws pro Tag bzw. 3849 Lkws pro Tag zu beschränken (vgl. Tab. 2). Allerdings ergab die Simulation, dass im Szenario 2004 bei Einführung des Umweltrechtesystems lediglich 409 Lkws pro Tag den Brenner überqueren. Dies entspräche einer jährlichen Belastung von 149285 Lkws, also nur knapp $10 \%$ des zulässigen Aufkommens.

\section{Abbildung 5}

Verkehrsaufkommen in den Szenarien

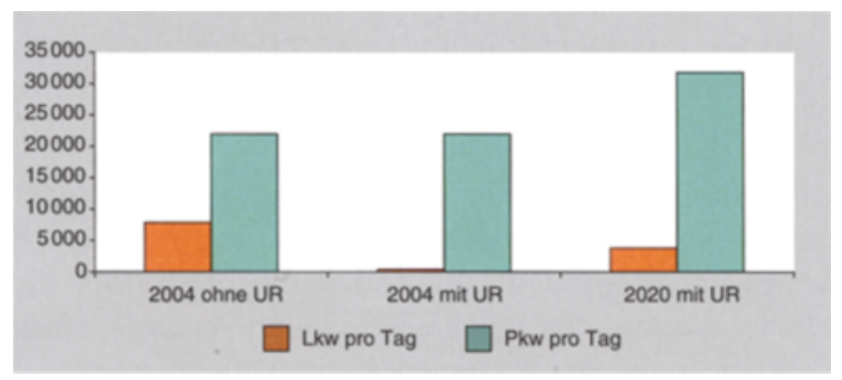

Für das Jahr 2020 ermittelte VACLAV, dass das Kontingent von 3849 Lkws pro Tag voll ausgeschöpft wird. Der Pkw-Verkehr steigt zwischen 2004 und 2020 erwartungsgemäß um über $44 \%$ auf 31795 Pkws pro Tag an. Der erheblich unrealistische Rückgang des Straßengüterverkehrsaufkommens in Szenario 2004 mit Umweltrechten kann zum einen mit der Festlegung eines zu strikten Reduktionsziels für 2004 oder zum anderen mit der Berechnung einer zu großen Transportnachfrage durch das Verkehrsmodul VACLAV begründet werden.

Dadurch, dass lediglich 3843 Lkws, also weniger als die Hälfte des von VACLAV berechneten täglichen LkwAufkommens von 7869 Lkws den Brenner überqueren dürfen, würden die Kosten einer Brennerüberquerung auf über 650 Euro steigen. ${ }^{3}$ Solch hohe finanzielle Belastungen hätten jedoch einen Rückgang des Lkw-Verkehrs über das notwendige Maß hinaus zur Folge, wie in Szenario 2 tatsächlich beobachtet. Daher können die im folgenden Kapitel untersuchten Auswirkungen auf die Belastungen durch Lärm, Stickoxide und Dieselruß für das Szenario 2 nur im Hinblick auf die Relevanz des Pkw-Verkehrs untersucht werden.

\section{Umweltwirkungen des Systems - Auswertung der Simulationsergebnisse}

Die Simulationsergebnisse zeigen, dass auf lange Sicht eine Steuerung des Straßengüterverkehraufkommens in ökologisch besonders sensiblen Regionen mit Hilfe handelbarer Umweltrechte möglich ist. Eine Reduzierung der Belastungen von Mensch und Umwelt durch Lärm, Stickoxide und Partikel kann somit umgesetzt werden.

Mit Hilfe von Immissionsberechnungen für Lärm, Stickoxide und Dieselruß sowie deren Verschneidung mit zuvor ermittelten raster-basierten Bevölkerungsdichten (Raster $250 \times 250 \mathrm{~m}$ ) konnten die Auswirkungen der einzelnen Szenarien analysiert werden. Das dem zugrunde liegende Konzept ist in Abbildung 6 am Beispiel der Berechnung und Auswertung der Lärmimmissionen dargestellt. 
Abbildung 6

Verwendetes Konzept zur Analyse der Umweltwirkungen

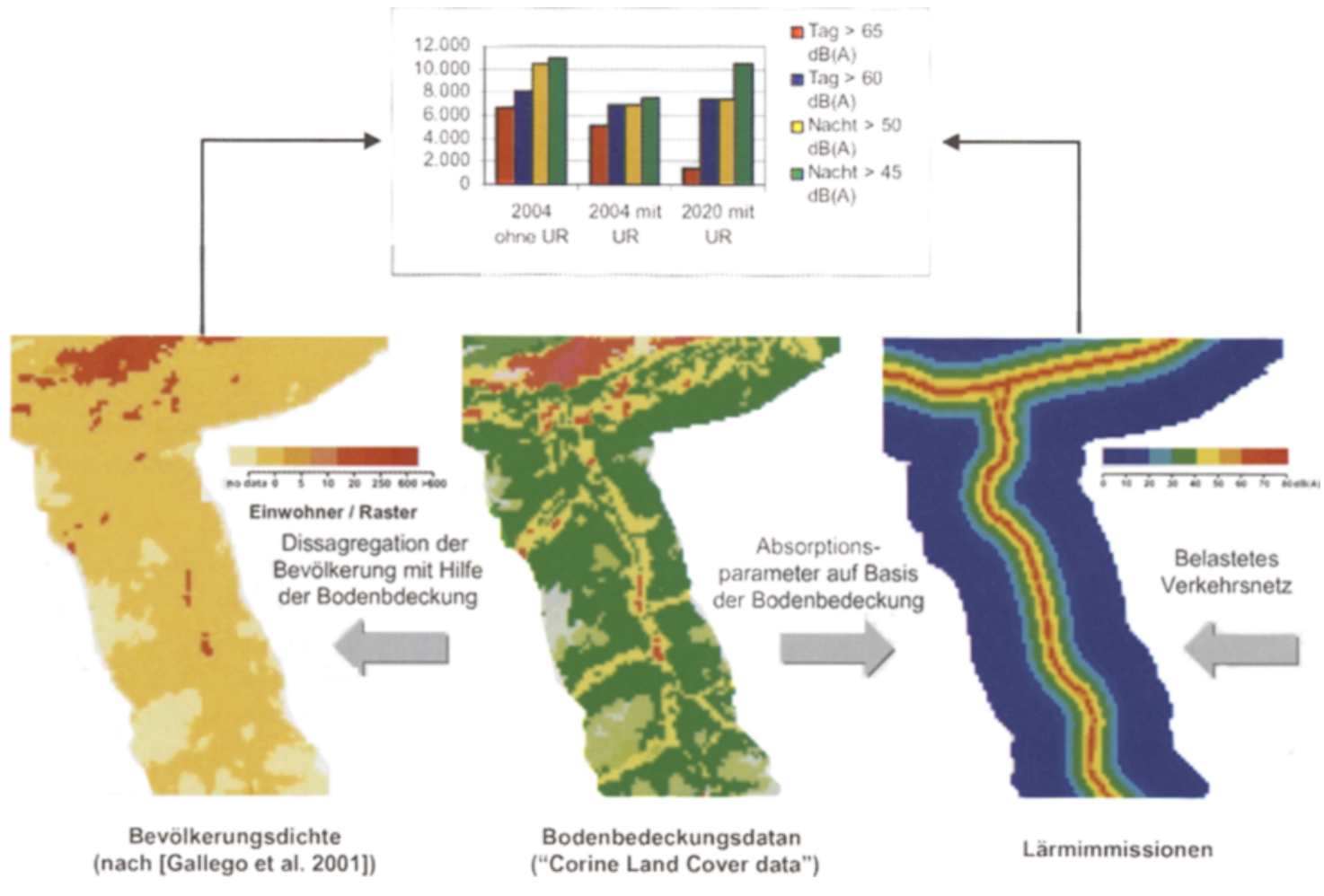

\section{Lärmbelastung}

Nach Einführung des Systems handelbarer Umweltrechte waren 2020 zirka $5 \%$ der in der betrachteten sensiblen Region lebenden Personen Lärmbelästigungen oberhalb der vom Umweltbundesamt gesetzten Zielwerte ausgesetzt. Der Anteil der tagsüber mit $65 \mathrm{~dB}(\mathrm{~A})$ belasteten Personen sank von $5 \%$ auf unter $1 \%$. Allerdings verdeutlicht Szenario II, dass nur durch die Ausdehnung des Systems auf den Pkw-Verkehr eine weitergehende Reduzierung der Lärmbelästigung möglich ist. Trotz einer Reduzierung des Lkw-Verkehrs um fast $95 \%$ liegt der Anteil der durch Lärm belasteten Personen 2004 im Szenario mit Umweltrechten um weniger als 0,5\% unter dem Anteil der 2020 durch Lärm belasteten Personen.

\section{Stickoxide}

Nach der Einführung des Systems handelbarer Umweltrechte ist mit einer erheblichen Reduktion der Stickstoffbelastungen zu rechnen. Die Betroffenheitsanalyse illustriert, dass nach Einführung des Umweltrechtesystems $2020 \mathrm{im}$ Mittel keine einzige Person mehr mit mehr als $65 \mu \mathrm{g} / \mathrm{m}^{3}$ Stickoxid belastet ist (vgl. Tabelle 3). Dass für das Szenario 2004 mit Umweltrech- ten ähnliche Werte ermittelt wurden verdeutlicht, dass der Hauptteil der Stickoxidemissionen auf den LkwVerkehr zurückzuführen ist.

Tabelle 3

Anteil der betroffenen Personen von Stickoxyd-Immissionen

\begin{tabular}{|l|c|c|}
\hline & $\begin{array}{c}\mathbf{2 4 h} \mathbf{M a x}> \\
\mathbf{6 5} \boldsymbol{\mu g} / \mathbf{m}^{\mathbf{3}}\end{array}$ & $\begin{array}{c}\mathbf{2 4 h} \mathbf{~ M i t t e l ~ > ~} \\
\mathbf{6 5} \mathbf{~} \mathrm{g} / \mathbf{m}^{\mathbf{3}}\end{array}$ \\
\hline 2004 ohne Umweltrechte & $6,5 \%$ & $1,4 \%$ \\
2004 mit Umweltrechte & $3,5 \%$ & $0,0 \%$ \\
2004 mit Umweltrechte & $5,5 \%$ & $0,0 \%$ \\
\hline
\end{tabular}

\section{Dieselruß}

Bezüglich der Belastungen durch Ruß schlägt die Arbeitsgruppe „Krebsrisiko durch Luftverunreinigungen“ des Länderausschusses für Immissionsschutz den Beurteilungsmaßstab von $1,5 \mu \mathrm{g} / \mathrm{m}^{3}$ vor (vgl. Länderausschuss für Immissionsschutz 1992). Das Umweltbundesamt strebt eine Reduzierung der Rußimmissionen auf $1,5 \mu \mathrm{g} / \mathrm{m}^{3}$ bis 2005 und $0,8 \mu \mathrm{g} / \mathrm{m}^{3}$ bis 2020 an (vgl. Gühnemann 1999). Wie Tabelle 4 zu entnehmen ist, könnte die Belastung durch Dieselruß im Szenario 2020 bei Einführung eines Systems handelbarer Um- 
weltrechte erheblich reduziert werden. So verringert sich der Anteil der Personen, die einer Belastung $>1,5$ $\mu \mathrm{g} / \mathrm{m}^{3}$ ausgesetzt sind, zwischen 2004 (ohne Umweltrechte) und 2020 (mit Umweltrechten) im 24-StundenMittel von 2,5\% auf $0,5 \%$ bzw. im 24-Stunden-Maximum von $8,6 \%$ auf $5,9 \%$. Zudem steigt die Anzahl der Personen, die Belastungen von im Mittel $<0,5 \mu \mathrm{g} / \mathrm{m}^{3}$ ausgesetzt sind, bis 2020 auf mehr als $95 \%$ an.

Tabelle 4

Anteil der betroffenen Personen von Dieselruß-Immissionen

\begin{tabular}{|l|c|c|}
\hline & $\begin{array}{c}\text { 2004 ohne } \\
\text { Umweltrechte }\end{array}$ & $\begin{array}{c}2020 \mathrm{mit} \\
\text { Umweltrechten }\end{array}$ \\
\hline Mittel $>1,5 \mu \mathrm{g} / \mathrm{m}^{3}$ & $2,5 \%$ & $0,5 \%$ \\
Max $>1,5 \mu \mathrm{g} / \mathrm{m}^{3}$ & $8,6 \%$ & $5,9 \%$ \\
Mittel $>0,5 \mu \mathrm{g} / \mathrm{m}^{3}$ & $92,8 \%$ & $95,6 \%$ \\
Max $>0,5 \mu \mathrm{g} / \mathrm{m}^{3}$ & $95,6 \%$ & $88,2 \%$ \\
\hline
\end{tabular}

Jedoch ist auch bei Betrachtung der Rußemissionen die Relevanz des Pkw-Verkehrs klar erkennbar. Selbst im Szenario 2004 mit Umweltrechten sind Belastungen von bis zu $0,5 \mu \mathrm{g} / \mathrm{m}^{3}$ entlang der parallel zur Al3 verlaufenden $B 182$ zu beobachten. Diese können aufgrund des Lkw-Fahrverbots im nebengelagerten Straßennetz oder ausschließlich durch den Personenverkehr verursacht worden sein.

\section{Gesamtergebnis}

Im betrachteten Fallbeispiel wurden nach Einführung der handelbaren Umweltrechte im Jahr 2020 die Belastungsziele bezüglich Lärm für $95 \%$ der betroffenen Personen erreicht. Die verkehrsbezogenen Stickoxidimmissionsgrenzwerte wurden gänzlich eingehalten. Zudem konnte eine erhebliche Reduzierung der Dieselrußemission verzeichnet werden, so dass 2020 weniger als $5 \%$ der im Untersuchungsraum betroffenen Personen Rußpartikelbelastungen von mehr als $0,5 \mu \mathrm{g}$ / $\mathrm{m}^{3}$ ausgesetzt waren.

Trotz dieser eindeutig positiven Effekte der Einführung des Umweltrechtesystems hob die Simulation aber auch zwei eher kritische Sachverhalte hervor: Zum einen verdeutlichte sie, dass die Festlegung eines adäquaten und realistischen Reduktionsziels entscheidend für den Erfolg des Systems ist. Ein zu einschränkendes Reduktionsziel kann aufgrund der Nichtausschöpfung der Kontingente vor allem in den ersten Laufzeitperioden zu erheblichen Effizienzverlusten führen.

\section{Zusammenfassung und Ausblick}

Dauerhafte Überlastungen der wichtigsten transeuropäischen Verkehrsachsen sowie die zunehmende Belastung durch Schadstoffe und Lärm beeinträchtigen Mensch und Umwelt. Vor allem in ökologisch besonders sensiblen und hoch belasteten Gebieten stößt somit der klassische verkehrspolitische Instrumentenmix an seine Grenzen und fällt vielmehr durch seine eher eingeschränkte ökologische Treffsicherheit und eine zweifelhafte Effektivität auf. Entsprechend herrscht weitgehend Konsens, dass neuartige verkehrspolitische Instrumente notwendig sind, die einerseits Angebot und Nachfrage bei der Verkehrsinfrastruktur wieder in Einklang bringen und andererseits die Belastungen für Mensch und Umwelt reduzieren.

Handelbare Umweltrechte zeichnen sich generell durch ein hohes Maß an ökologischer Treffsicherheit wie auch ökonomischer Effizienz aus. Sie erreichen so ein hoheitlich festgelegtes Umweltziel zu gesamtwirtschaftlich minimalen Kosten. Das hier vorgestellte und in seinen Auswirkungen am Beispiel der Brennerregion simulierte System handelbarer Umweltrechte für den Straßengüterverkehr zeigt, dass solche Systeme ein vielversprechendes verkehrspolitisches Instrument zur Reduzierung verkehrsbedingter Belastungen in sensiblen und hoch belasteten Regionen darstellen. Ihr möglicher Einsatz sollte daher in die Diskussion potenzieller neuartiger politischer Maßnahmen integriert werden.

\section{Anmerkungen}

(1)

„Up- und Downstream“-Prozesse bezeichnen Schäden, die zum Beispiel bei der Herstellung von Fahrzeugen („upstream“) sowie bei deren Verschrottung (",downstream“) entstehen.

(2)

Im Jahr 2003 überquerten $1574000 \mathrm{Lkw}$ pro Jahr = $4312 \mathrm{Lkw}$ pro Tag den Brenner (vgl. TL 2004, S.10).

(3)

p2004 $=322 * \ln (7868)-2234=654,52$ Euro (entnommen aus dem in (Eßer 2004, Anhang G) dargestellten Berechnungsverfahren)

\section{Literatur}

Baumol, W., Oates, W.: The theory of environmental policy. Cambridge 1998

Bickel, P., Friedrich, R.: Was kostet uns die Mobilität? Externe Kosten des Verkehrs. - Berlin 1994. 
Bundesamt für Umwelt, Wald und Landschaft - BUWAL: Nabel - Das Nationale Beobachtungsnetz für Luftschadstoffe (www.buwal.ch/publikat/d, 2004)

Eßer, A.: Die Konzeption eines Systems handelbarer Umweltrechte für den Straßengüterverkehr in ökologisch besonders „sensiblen Regionen". Diplomarbeit Univ. Karlsruhe (TH) 2004

Endres, A., Holm-Müller, K. : Die Bewertung von Umweltschäden - Theroie und Praxis sozioökonomischer Verfahren. - Stuttgart 1998

Europäische Kommission: European Union - Energie \& Transport in Figures. - Brüssel 2003

Gallego, J., Peedell, S.: Using land cover information to map population density. Working Paper. Joint research Center, Ispra (Italien) 2001

Geisendorf, S.: Das Problem der Internalisierung externer Kosten des Straßengüterverkehrs am Beispiel von $\mathrm{CO}_{2}$-Zertifikaten. EURES discussion paper 23 (1994)

Schütte, C.: Road-pricing in der Praxis. - TU Berlin 1998

Gühnemann, A.: Methods for strategic environmental assessment of transport infrastructure plans. - Univ. Karlsruhe (TH) 2004.

Hansjürgens, B., Gangelmann, F.: Das Handelssystem im europäischen $\mathrm{CO}_{2}$-Emissionsrechtemarkt - Vorschläge zur Institutionellen Ausgestaltung in Deutschland. - Leipzig-Halle 2003. = UFZ Diskussionspapier 12/2003

INFRAS; IWW : External Costs of Transport - Update Study. - Internationaler Eisenbahnverband (UIC), Paris 2004

Koschel, H.; Brockmann, K.; Schmidt, T.; Stronzik, M.; Bergmann, H.: Handelbare $\mathrm{SO}_{2}$-Zertifikate für Europa: Konzeption und Wirkungsanalyse eines Modellvorschlags. - Heidelberg 1998

Länderausschuss für Immissionsschutz: Beurteilungsmaßstäbe zur Begrenzung des Krebsrisikos durch Luftverschmutzung. In: Krebsrisiko durch Luftverunrienigungen. Hrsg.: Ministerium für Umwelt, Raumordnung und Landwirtschaft des Landes Nordrhein-Westfalen. - Düsseldorf 1992

Lebek, H.: Auswirkungen der Maut auf Verkehrsgewerbe und Frachtenhöhe. In: Maut Management. Hrsg.: Deutscher VerkehrsVerlag. - Hamburg 2003, S. 33-46

Kraetzschmer, D.; Hanusch, M.; Rothengatter, W.; Schmedding, D.; Schade, W. u.a.: Transport-related impacts and instruments for sensitive areas (http://europa.eu.int/comm/ environment/air/sat.htm, 2004)
Schade, W.: Strategic Sustainability Analysis: Concept and application for the assessment of European Transport Policy. - Univ. Karlsruhe (TH) 2004

Schoch, M.: Methoden zur Bestimmung der geeigneten räumlichen Aggregation in Personenfernverkehrsmodellen. - Univ. Karlsruhe (TH) 2004

Schmedding, D.; Schade, W.: The development and implementation of a model to assess traffic-related soot-particle and nitrogen oxide emissions in regions. In: Air Pollution XIII. Hrsg.: Brebbig, C. A. - Cordoba 2005, S. 327-336

TEN-STAC: Trans-European Transport Network-Scenarios, Traffic Forecasts and Analyses of Corridors on the Trans-European Transport Network, Deliverable D1 (www.nea.nl/ten-stac, 2004)

Tiroler Landesregierung: Verkehr in Tirol. Bericht 2003 (www.tirol.gv.at/themen/verkehr/service/verkehrsbericht, 2004)

Troge, A.: Verkehr: Ausnahmebereich für Emissionshandelsstrategien. In: Verkehr und Umwelt: Gesamtwirtschaftliche Bewertung von Maßnahmen zur Verringerung von Luftschadstoffemissionen des Verkehrs. Hrsg.: DVWG. - Bergisch-Gladbach 1993. = DVWGSchriftenreihe B 166, S. 6-32

Dipl.-Wi.-Ing. Anke Eßer

Universität Karlsruhe (TH)

Institut für Industriebetriebslehre und

Industrielle Produktion (IIP)

Hertzstraße 16

76187 Karlsruhe

E-Mail: anke.esser@wiwi.uni-karlsruhe.de

Dipl.-Wi.-Ing. David Schmedding

Universität Karlsruhe (TH)

Institut für Wirtschaftspolitik und

Wirtschaftsforschung (IWW)

Kaiserstraße 12

76131 Karlsruhe

E-Mail: schmedding@iww.uni-karlsruhe.de

\footnotetext{
Hinweis:

Dieser Beitrag wurde im Rahmen des Werner-ErnstPreis-Wettbewerbs des Förderkreises für Raum- und Umweltforschung e. V. (FRU) 2005 zum Thema „Grenzenloser Verkehr? - Verkehr an Grenzen!“ mit dem 2. Preis ausgezeichnet.
} 